\title{
ARTICLE
}

\section{An in vivo strategy to counteract post-administration anticoagulant activity of azido-Warfarin}

\author{
Sylvain Ursuegui ${ }^{1, \star}$, Marion Recher ${ }^{1, \star}$, Wojciech Krężel $2,3,4,5,6$ \& Alain Wagner ${ }^{1}$
}

Drugs, usually long acting and metabolically stable molecules, might be the source of adverse effects triggered by complex drug interactions, anaphylaxis and drug-induced coagulopathy. To circumvent this growing drug safety issue, we herein investigate the opportunity offered by bio-orthogonal chemistry for in vivo drug neutralization. We design a small-molecule anticoagulant drug (Warfarin) containing an azide group that acts as a safety pin. It allows drug deactivation and restoration of physiological coagulation via in vivo click reaction with a suitable cyclooctyne-based neutralizing agent. In this strategy, the new molecule formed by reaction of the drug and the antidote is deprived of biological activity and prone to fast renal clearance. This 'Click \& Clear' approach lays ground for new strategies in designing drugs with switchable biophysical properties.

\footnotetext{
${ }^{1}$ Laboratory of Functional Chemo-Systems (UMR 7199), Labex Medalis, University of Strasbourg, 74 Route du Rhin, 67401 IIIkirch-Graffenstaden, France. ${ }^{2}$ Institut de Génétique et de Biologie Moléculaire et Cellulaire, , 67404 Illkirch, France. ${ }^{3}$ Institut de la Santé et de la Recherche Médicale, U964, 67404 IIIkirch, France. ${ }^{4}$ Centre National de la Recherche Scientifique, UMR7104, 67404 IIlkirch, France. ${ }^{5}$ Université de Strasbourg, 67404 IIlkirch, France.

${ }^{6}$ Fédération de Médecine Translationnelle de Strasbourg, 67404 Illkirch, France. *These authors contributed equally to this work. Correspondence and requests for materials should be addressed to W.K. (email: krezel@igbmc.fr) or to A.W. (email: alwag@unistra.fr).
} 
O ptimization of absorption, distribution, metabolism and excretion profile to allow the most efficient targeting of the effector site is an essential step in the course of drug development. Indeed, once the drug has been administered and after its entry into the systemic circulation, it undergoes complex distribution, metabolism and excretion processes. Consequently, the ability of the chemist to tweak molecule structure to optimize its absorption, distribution, metabolism and excretion profile without losing pharmacological activity or in vivo specificity accounts for a large part of a drug's therapeutic efficacy.

However, such optimized drugs, usually long acting and metabolically stable molecules, might also be the source of some adverse effects. Among classical examples of iatrogenic accidents, triggered by molecular therapy, are complex drug interactions, anaphylaxis and drug-induced coagulopathy. To circumvent this growing drug safety issue, researchers have started to develop dual drug-antidote strategies. The most successful system currently used in the clinic consists of administering a $\gamma$-cyclodextrin bearing a hydrophobic core and a hydrophilic crown tailored to scavenge the muscle relaxants rocuronium and vecuronium during general anaesthesia ${ }^{1-4}$. Another approach focuses on the development of a novel type of aptamer-based anticoagulant that can be neutralized with antisense oligonucleotides ${ }^{5-8}$, porphyrines ${ }^{9,10}$ or positively charged oligomers ${ }^{11}$. The system REG 1, comprising an aptamer as a reversible antagonists of coagulation factor IXa and its complementary oligonucleotides as antidote, was taken into clinic trials but, to the best of our knowledge, has been halted in phase III (ref. 12). Both of these approaches rely on specific non-covalent interaction to scavenge the exogenous compound preventing its biological effect without removing it from the organism.

To address the issue of drug neutralization and clearance, we have investigated the opportunity offered by in vivo bioorthogonal chemistry. Our idea was to design a small-molecule drug containing an azide group that would act as a safety pin, allowing drug deactivation and clearance via an in vivo click reaction with a suitable strained cyclooctyne-containing neutralizing agent. In this strategy, the new molecule formed by reaction of the drug and the antidote should ideally be deprived of any biological activity and quickly cleared from the bloodstream via renal excretion.

Previously, click chemistry has been used to trace proteins bearing azido amino acids and lipids ${ }^{13-16}$. This chemistry has also been demonstrated to be efficient for labelling azidosugars in the physiologically relevant context of a mouse $\mathrm{e}^{17-19}$, and more recently for pre-targeted tumour imaging in living mice ${ }^{20}$. These results establish the feasibility of using in vivo bioorthogonal chemical reaction in living organisms when one reactant is targeted to specific tissues (via a labelled antibody or a polymer $)^{21}$. In pioneering work, Rossin et al. ${ }^{22}$ developed a novel 2-tetrazine-functionalized clearing agents that enable the rapid reaction with and the removal of a TCO-tagged antibody from blood. This clearing agent led to 125-fold improvement of the $\mathrm{Ab}$ tumour-to-blood ratio $3 \mathrm{~h}$ after tetrazine injection. This was achieved through rapid reaction of clearing agents with the $\mathrm{mAb}$ in blood to remove it from the circulation and concentrate it in the liver or spleen without blocking the tumour-bound $\mathrm{mAb}$.

\section{Results}

General. Here we demonstrate that click chemistry can proceed in the vasculature at the level of the whole organism and can be used for targeted drug deactivation and clearance. We study the neutralization of 3-(1-(4-azidophenyl)-3-oxobutyl)-2H-chromen2-one $\left(\mathrm{WN}_{3}, 2\right)$, an isosteric analogue of Warfarin (W), a well-known anti-vitamin $\mathrm{K}$ anticoagulant agent, by click reaction with an optimized soluble analogue of BiCyclo[6.1.0] Nonyne (BCN-peg 6 -OH 9, Fig. 1).

$\mathrm{WN}_{3}$ (2) and clearing cycloalkyne (9) derivatives synthesis. The introduction of an azide moiety on warfarin derivative 1a should maintain the anticoagulant activity. Accordingly, whereas studies have shown that substitution on the 4-hydroxycoumarin motif resulted in a significant reduction of the anticoagulant activity ${ }^{23}$, the substitution on the phenyl moiety is innocuous to the warfarin's anticoagulant activity. Indeed acenocoumarol $\mathbf{1 b}$, $4^{\prime}$-nitro warfarin analogue, is currently used in clinic. For this

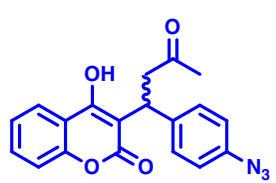

Anticoagulant drug $\mathrm{WN}_{3}(2)$

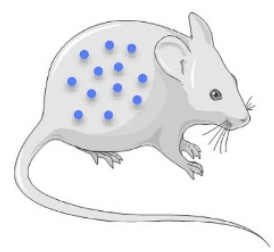

Mice treated with anticoagulant drug
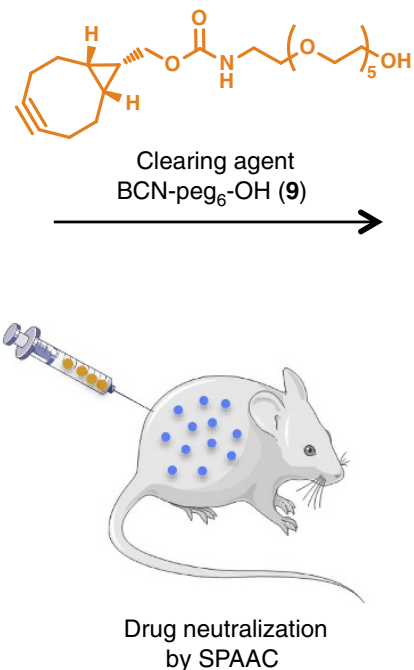

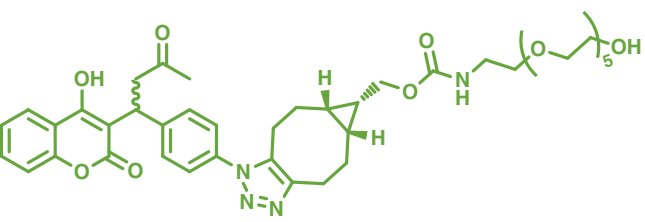

Inactived clicked drug clicked product (10)

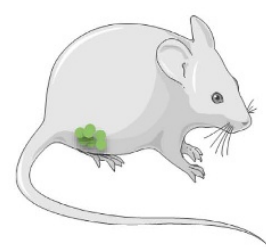

Mice with normal coagulation

Figure 1 | 'Click and Clear' strategy. Biological inactivation and fast clearance of a circulating drug, Warfarin- $\mathrm{N}_{3} \mathbf{2}$, by in vivo Strain Promoted Alkyne Azide Cycloaddition (SPAAC) reaction. A mouse submitted to anticoagulant therapy $\left(\mathrm{WN}_{3}, \mathbf{2}\right)$ is treated with a clearing agent (BCN-peg $\left.-\mathrm{OH}, \mathbf{9}\right)$ prone to react with the anticoagulant drug. In vivo bio-orthogonal reaction between circulating $\mathrm{WN}_{3} \mathbf{2}$ and $\mathrm{BCN}$-peg $-\mathrm{OH} \mathbf{9}$ leads to the formation of an inactivated compound $\mathbf{1 0}$ which is readily cleared from the bloodstream, restoring normal coagulation activity. The pictures of mice and syringe have been downloaded from Servier Medical Art Database which provides these illustrations through the Creative Commons license (https://creativecommons.org/licenses/by/ $3.0 /)$. 
a

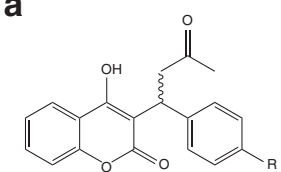

1a, $\mathrm{R}=\mathrm{H}$ warfarin

b, $\mathrm{R}=\mathrm{NO}_{2}$ acenocoumaro b

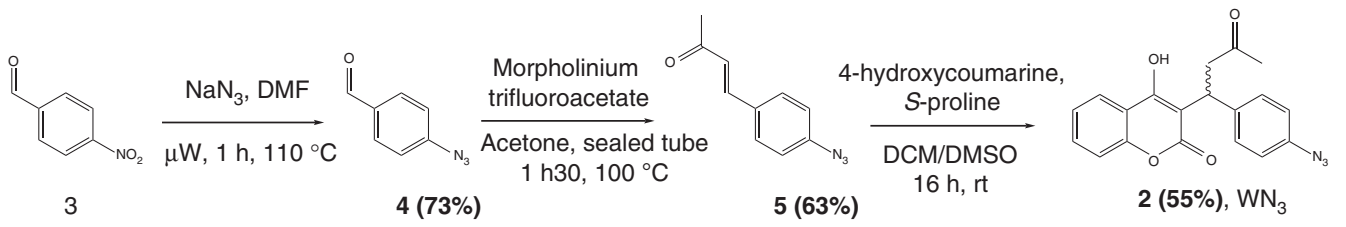

C
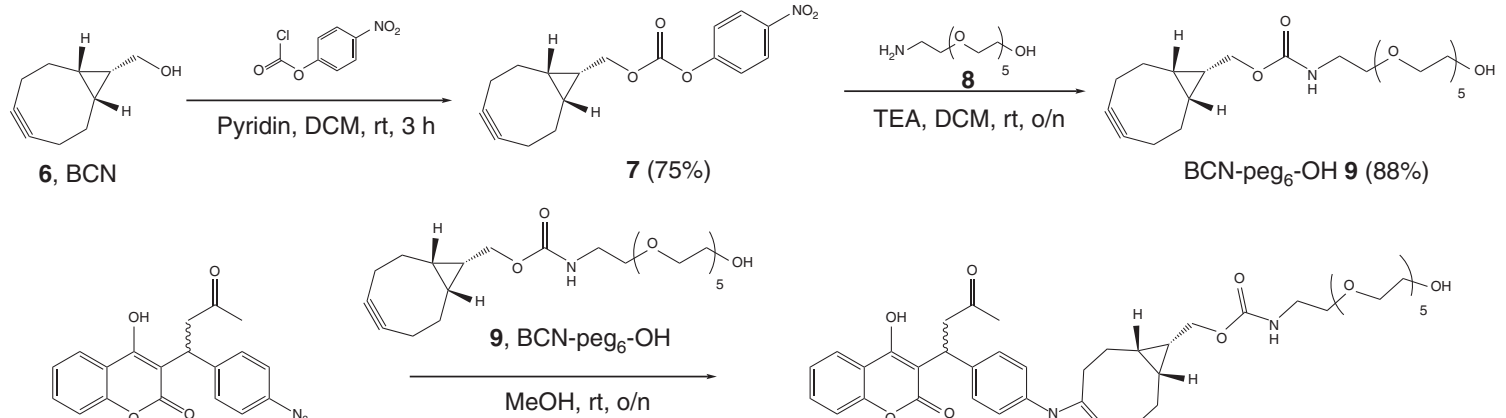

2. $\mathrm{WN}_{3}$

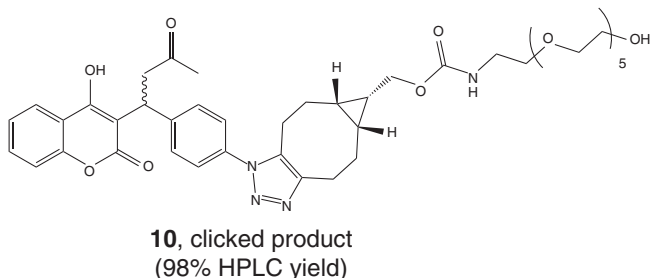

Figure 2 | Synthesis of chemical compounds. (a) Structure of Warfarin $\mathbf{1 a}$ and acenocoumarol $\mathbf{1 b}$ anticoagulant drugs. (b) Synthesis of $W N_{3} \mathbf{2}$ anticoagulant analogue. (c) Synthesis of the clearing agent: $\mathrm{BCN}-\mathrm{peg}_{6}-\mathrm{OH}$ 9. (d) Synthesis of the clicked product resulting from WN 2 and $\mathrm{BCN}-\mathrm{peg}_{6}-\mathrm{OH}$ 9 reaction.

reason we decided to introduce the azide group in $4^{\prime}$ position of the warfarin phenyl ring. The synthesis of $\mathrm{WN}_{3} 2$ was achieved as illustrated in Fig. 2b.

To circumvent the low solubility of BCN, which could hamper its administration and activity in vivo, we synthesized a neutralizing agent $\mathrm{BCN}-\mathrm{peg}_{6} \mathrm{OH} 9$ derivative. This analogue of $\mathrm{BCN}$ is soluble enough to reach high concentration in the plasma and can be efficiently detected in plasma at low concentrations. The synthesis of $\mathrm{BCN}-$ peg $_{6}-\mathrm{OH} 9$ was achieved as depicted in Fig. 2c.

The reactivity of $\mathrm{WN}_{3} 2$ was evaluated towards $\mathrm{BCN}-\mathrm{peg}_{6}-\mathrm{OH}$ 9 in plasma. Since interactions of plasma proteins with reagents could indirectly affect our measures, we first, showed that all forms of warfarin (1a, 2 and 10) were completely extracted from plasma using acetonitrile-the procedure used for the subsequent kinetics studies (Supplementary Fig. 7). Then kinetic experiments were recorded in plasma using equimolar $(100 \mu \mathrm{M})$ concentrations of both compounds. $\mathrm{WN}_{3} 2$ was found to react efficiently with BCN-peg6-OH 9 in plasma without formation of any by-product (Supplementary Fig. 11) with a rate constant of $6.8 \pm 1.8 \mathrm{M}^{-1} \mathrm{~s}^{-1}$ (Supplementary Fig. 12).

The stability of $\mathrm{WN}_{3} 2$ and $\mathrm{BCN}-\mathrm{peg}_{6}-\mathrm{OH} 9$ in plasma was then assessed. $\mathrm{WN}_{3} 2$ was found to be stable when kept at $37^{\circ} \mathrm{C}$ in the plasma or as dried powder for more than $24 \mathrm{~h}$ without particular precaution. $\mathrm{WN}_{3} 2$ stability in plasma was also assessed by recording the kinetic of neutralization with BCN-peg 6 - $\mathrm{OH} 9$ after incubation of a solution of $\mathrm{WN}_{3} 2$ aged in plasma at $37^{\circ} \mathrm{C}$ for $15 \mathrm{~h}$. Under this condition a reaction rate constant of $5.1 \pm 0.8 \mathrm{M}^{-1} \mathrm{~s}^{-1}$ was measured (Supplementary Fig. 13), close to that observed in the control experiment using fresh solution of reagents $\left(6.8 \pm 1.8 \mathrm{M}^{-1} \mathrm{~s}^{-1}\right.$, Supplementary Fig. 12$)$. BCN is known to be a reactive scaffold with limited stability in biological media. We thus decided to evaluate the effect of the time of residence of $\mathrm{BCN}-$ peg $_{6}-\mathrm{OH} 9$ in the plasma at $37^{\circ} \mathrm{C}$ on its ability to neutralize $\mathrm{WN}_{3}$ 2. As expected, we found that after $15 \mathrm{~h}$ of incubating $\mathrm{BCN}-$ peg $_{6}-\mathrm{OH} 9$ in human plasma at $37^{\circ} \mathrm{C}$, kinetic constant decreased with time from $6.8 \pm 1.8$ to $2.4 \pm 0.8 \mathrm{M}^{-1} \mathrm{~s}^{-1}$ (Supplementary Fig. 14). This suggests that $\mathrm{BCN}-$ peg $_{6}-\mathrm{OH} 9$ in plasma shows a suitable reactivity versus stability balance during limited time-window, yet sufficiently long to be suitable for in vivo experiments (compare Fig. 7).

$\mathbf{W N}_{3}$ (2) anticoagulant activity. To evaluate the anticoagulant activity of the synthesized compounds, we measured the prothrombin time (PT), which is the standard parameter used in clinics for the evaluation of coagulation process in patients treated with anti-vitamin $\mathrm{K}$ compounds. To this end, we first validated and optimized this technique for the measurement of anticoagulant activity of pharmacological treatments in mice. In agreement with previous reports ${ }^{24}$, the basal PT was found to range between 13 and $16 \mathrm{~s}$ (14.8 $\pm 0.2 \mathrm{~s}$, Supplementary Fig. 15), indicating thus that any higher PT will reflect an anticoagulant activity.

We then confirmed the activity of $\mathrm{WN}_{3} 2$ compared to the parent compound Warfarin 1a. Two per os administrations of Warfarin $\left(10 \mathrm{mg} \mathrm{kg}^{-1}\right)$ led to a significant increase of PT values reaching $451.2 \pm 135.1 \mathrm{~s}(P<0.01$, as compared to $14.8 \pm 0.2$ in control untreated mice; Supplementary Fig. 15). Similarly, significant anticoagulant effect was also observed after per os administration of $\mathrm{WN}_{3} 2(403.0 \pm 207.2 \mathrm{~s}, \quad P<0.05$; Supplementary Fig. 15). However, some samples in each of the two treatment groups did not coagulate within $15 \mathrm{~min}$ of the PT cut-off period, leading thus to high variability of experimental data and reflecting possible effect of the high dose, but also differences in absorption and metabolism of both compounds. To reduce such variability, we focused on $\mathrm{WN}_{3} 2$ and used lower doses and a different administration method. To this end, mice were injected (intraperitoneal (IP)) twice at a 24 -h interval with different doses of $\mathrm{WN}_{3} 2$ and were killed $6 \mathrm{~h}$ after the last injection. We observed a dose-dependent increase of PT reaching $25.9 \pm 2.8 \mathrm{~s}$ for $1 \mathrm{mg} \mathrm{kg}^{-1}$ and $58.5 \pm 9.7$ for $2 \mathrm{mg} \mathrm{kg}^{-1}$ (Fig. 3a). This dose/response effect displayed low variability and was supported by significant effect of the dose $(F[2,15]=11.8$, $P<0.001$; one-way analysis of variance (ANOVA)). In the next round of experiments, we evaluated optimal delay after the last 

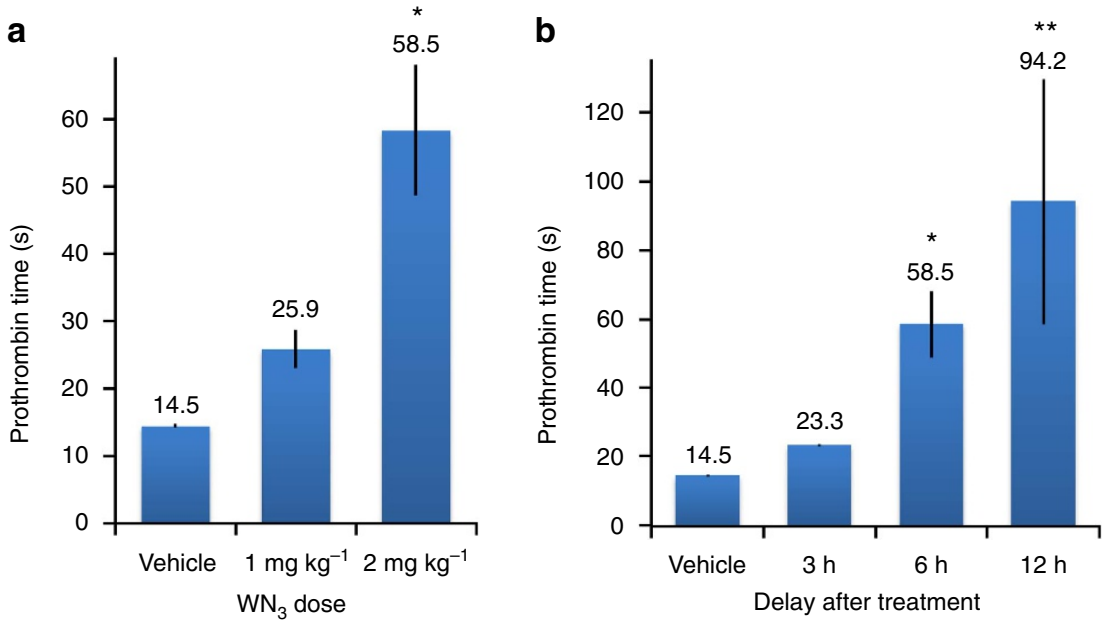

Figure 3 | In vivo biological activity of $\mathbf{W N}_{\mathbf{3}}$ (2) analogue. (a) PT depends from the $\mathrm{WN}_{3} \mathbf{2}$ dose delivered by IP injection. Dose/response curve of $W_{3} \mathbf{2}$ was measured $6 \mathrm{~h}$ after the second injection of 1 and $2 \mathrm{mg} \mathrm{kg}^{-1}$ of $\mathrm{WN}_{3} \mathbf{2}$ and compared to vehicle-treated mice (two IP injections, $n=4$ ). (b) PT depends on the delay after treatment. $\mathrm{WN}_{3} \mathbf{2}\left(2 \mathrm{mg} \mathrm{kg}^{-1}\right)$ was administered twice with a 24-h interval by IP injection and PT was measured 3, 6 and $12 \mathrm{~h}$ ( $n=4-6 /$ group) after the second treatment and compared to vehicle-treated mice. Statistical differences identified with protected least significant difference (PLSD) Fischer test were indicated as: ${ }^{\star} P<0.05,{ }^{\star \star} P<0.01$ as compared with vehicle-treated mice. Error bars indicate s.e.m. values.

treatment to obtain significant and reproducible anticoagulant effect. After two IP injections of $2 \mathrm{mg} \mathrm{kg}^{-1}$ of $\mathrm{WN}_{3} 2$, mice were killed at different time points for blood collection and PT measurements. A time dependence of anticoagulant activity was supported by significant effect of the test time after injection on PT measurements $(F[3,16]=4.8, P<0.05$; one-way ANOVA). $3 \mathrm{~h}$ after the last treatment, a slight but not significant increase of PT was observed $(23.3 \pm 0.3 \mathrm{~s}$ as compared to $14.5 \pm 0.5 \mathrm{~s}$ in control group; $P=0.7)$. This increase was enhanced and significant at $6 \mathrm{~h}$ $(58.5 \pm 9.7 ; P<0.05)$ and $12 \mathrm{~h}(94.2 \pm 35.6 \mathrm{~s} ; P<0.01)$, reflecting clearly a time-dependent activity of $\mathrm{WN}_{3} 2$ (Fig. 3b). Although $12 \mathrm{~h}$ after treatment, biological activity was the highest, it was associated with an important biological variability reflected by a high s.e.m. As a result of these analyses, we retained for further functional studies an IP injection of $2 \mathrm{mg} \mathrm{kg}^{-1}$ and PT measurement after $6 \mathrm{~h}$ as optimal experimental conditions to investigate in vivo drug inactivation, providing significant, $\sim 3.5$-fold increase of anticoagulant activity (as compared to the control group) with low variability. Moreover, this dose of $\mathrm{WN}_{3} 2$ corresponds to doses of Warfarin 1a used in clinical conditions as Warfarin dose used in human varies to attain 2-4-fold increase of PT. We also confirmed that the prolongation of PT after $\mathrm{WN}_{3} 2$ or Warfarin 1a treatment results in the concomitant decrease of the three-major vitamin K-dependent clotting factors, II, VII and $\mathrm{X}$ as revealed by PT measures using dedicated clotting factor assays (Supplementary Figs 19 and 20).

Cycloalkyne (9) and clicked product (10) biological activities. Prior to testing the $\mathrm{WN}_{3} 2$ inactivation by click reaction in vivo, we investigated whether SPAAC modification of $\mathrm{WN}_{3}$ carried out in vitro affects anticoagulant activity of $\mathrm{WN}_{3} 2$. The SPAAC modified $\mathrm{WN}_{3} 10$ did not display any anticoagulant activity (12.7 s $\pm 0.4 ; P=0.6$, as compared to $14.5 \pm 0.5 \mathrm{~s}$ in IP vehicle treatment, Fig. $4 \mathrm{f}$ ) when administrated by two IP injections at the dose of $4.6 \mathrm{mg} \mathrm{kg}^{-1}$ corresponding to 1 equimolar quantity of $\mathrm{WN}_{3}$ at $2 \mathrm{mg} \mathrm{kg}^{-1}$. Importantly, an IP or sub-cutaneous (SC) injection of BCN-peg $6_{6}-\mathrm{OH} 9\left(26 \mathrm{mg} \mathrm{kg}^{-1}\right.$ corresponding to 10 equimolar quantity of $2 \mathrm{mg} \mathrm{kg}^{-1}$ of $\mathrm{WN}_{3}$ ), did not affect anticoagulant activity in mice (IP: $12.0 \pm 0.4 \mathrm{~s}, P>0.2$ and SC: $13.0 \pm 0.7 \mathrm{~s}, P>0.9$ as compared to vehicle-treated mice). Further increasing the $\mathrm{BCN}-$ peg $_{6}-\mathrm{OH} 9$ dose to $52 \mathrm{mg} \mathrm{kg}^{-1}$,

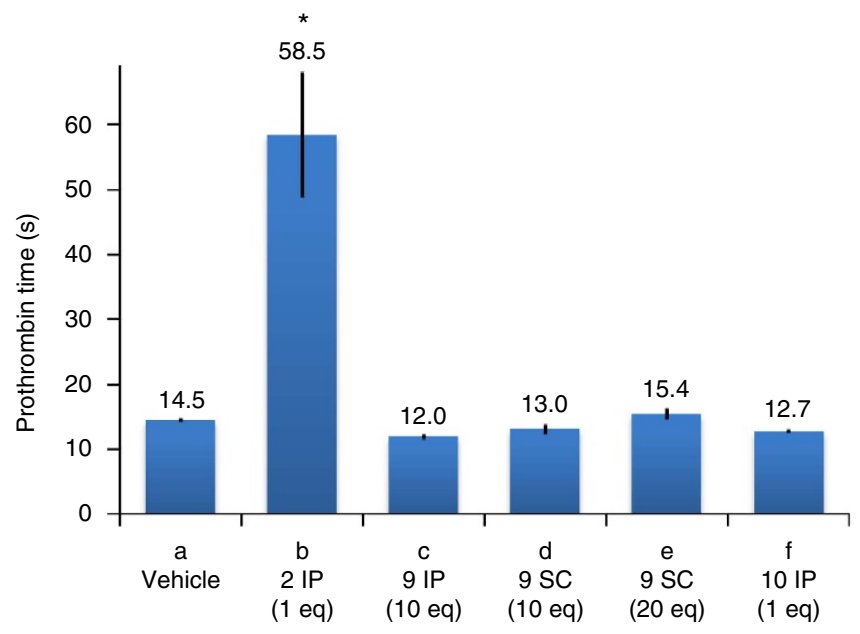

Figure 4 | Anticoagulant activity of $\mathrm{BCN}-\operatorname{peg}_{6}-\mathrm{OH}(9)$ and in vitro-clicked product (10). PT values were determined for different compounds following two IP or SC injections with a 24-h interval, as follows: (a) vehicle IP injections to establish control group of mice, $n=4$. (b) $W_{3} 2$ IP injections ( $2 \mathrm{mg} \mathrm{kg}^{-1}, n=7$ ). (c) BCN-peg 6 - $\mathrm{OH} 9 \mathrm{IP}$ injections (26 mg kg ${ }^{-1}$ (10 eq), $n=4)$. (d) BCN-peg $6-\mathrm{OH} 9 \mathrm{SC}$ injections ( $\left.26 \mathrm{mg} \mathrm{kg}^{-1}(10 \mathrm{eq}), n=4\right)$.

(e) $\mathrm{BCN}-$ peg $_{6}-\mathrm{OH} 9 \mathrm{SC}$ injections $\left(52 \mathrm{mg} \mathrm{kg}^{-1}(20 \mathrm{eq}), n=4\right)$. (f) Clicked product $10 \mathrm{IP}$ injections $\left(4.6 \mathrm{mg} \mathrm{kg}^{-1}(1 \mathrm{eq}), n=4\right) .{ }^{\star} P<0.1, \mathrm{PLSD}$ Fisher comparison with vehicle-treated mice. Error bars indicate s.e.m. values.

corresponding to 20 equimolar quantity of $\mathrm{WN}_{3} 2$ at $2 \mathrm{mg} \mathrm{kg}^{-1}$, did not alter blood coagulation as analysed for SC administration $(15.3 \pm 0.8, P=0.9)$. Such observations indicate that if click reaction proceeds in vivo it should induce the inactivation of $\mathrm{WN}_{3} 2$ anticoagulant activity and the decrease of the PT.

As all measures of $\mathrm{BCN}-$ peg $_{6}-\mathrm{OH} 9$ activity were performed in non-stimulated conditions when PT values are naturally low, we could not exclude the possibility that some endogenous procoagulant activity of $\mathrm{BCN}-\mathrm{peg}_{6}-\mathrm{OH} 9$ would be masked by the 'floor effect' of PT measurements. To address this point, we tested activity of BCN-peg 6 - $\mathrm{OH} 9$ in mice in which PT was increased by treatment with Warfarin 1a (which does not bear an azide group) and thus which should not be sensitive to the click reaction. As 
a

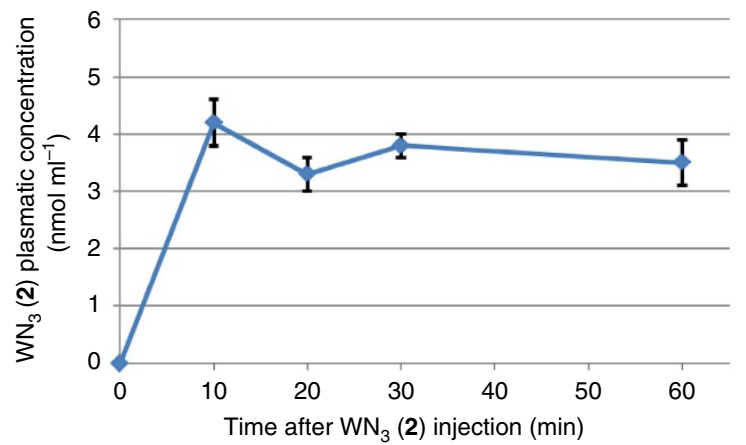

b

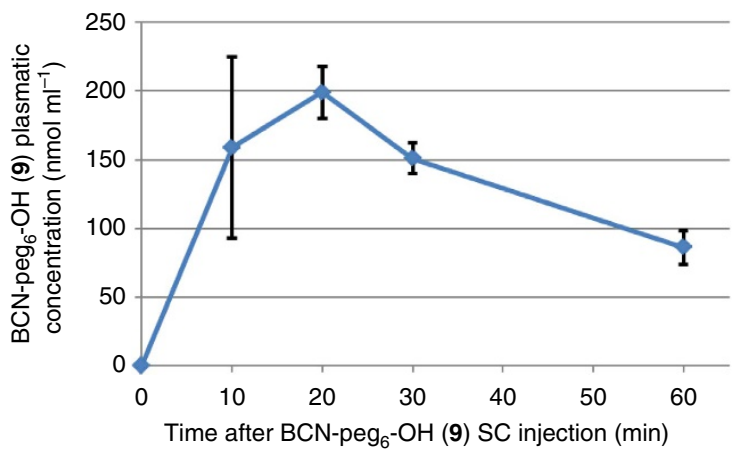

c

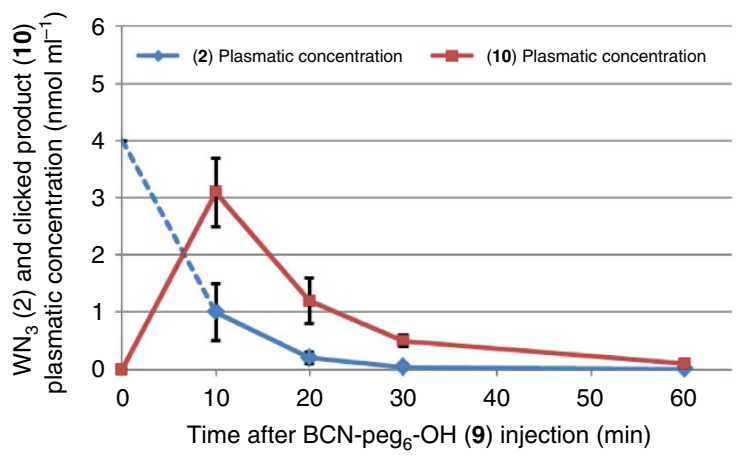

d

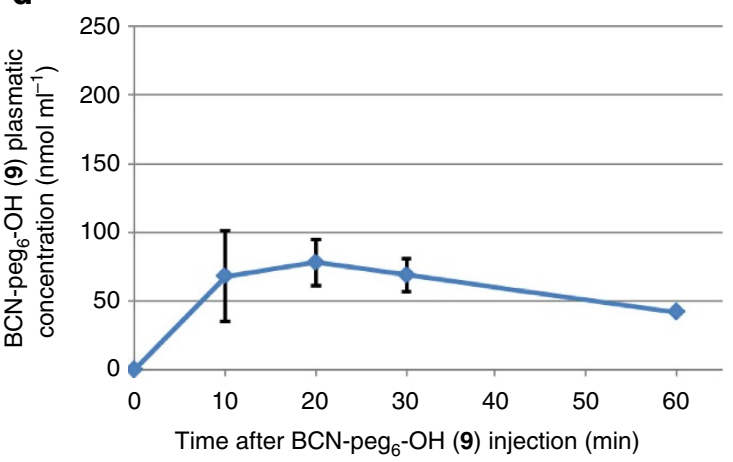

Figure 5 | LC-MS/MS analysis in plasma. (a,b) $\mathrm{WN}_{3}(\mathbf{2})$ and $\mathrm{BCN}-\mathrm{peg}_{6}-\mathrm{OH}(\mathbf{9})$ pharmacokinetics. (a) Plasma concentration of $\mathrm{WN}_{3} \mathbf{2}$ after IP injection $\left(2 \mathrm{mg} \mathrm{kg}^{-1}, n=4\right)$. (b) Plasmatic concentration of BCN-peg $6-\mathrm{OH} \mathbf{9}$ after SC injection (52 $\left.\mathrm{mg} \mathrm{kg}^{-1}, 114.4 \mu \mathrm{mol} \mathrm{kg}-1, n=4\right)$. (c,d) LC-MS/MS analysis of in vivo click reaction. $\mathrm{WN}_{3} \mathbf{2}\left(2.0 \mathrm{mg} \mathrm{kg}^{-1}, 5.72 \mu \mathrm{mol} \mathrm{kg}{ }^{-1}, 1 \mathrm{eq}\right)$ was administered by IP injection followed by the BCN-peg $6^{-} \mathrm{OH} \mathbf{9}$ delivery $\left(52 \mathrm{mg} \mathrm{kg}^{-1}, 114.4 \mu \mathrm{mol} \mathrm{kg}-1,20\right.$ eq) by SC injection 5 min later. Blood collection was performed after $\mathrm{BCN}-\mathrm{peg}_{6}-\mathrm{OH} \mathbf{9}$ injection to determine $\mathrm{WN}_{3} \mathbf{2}$, $\mathrm{BCN}-$ peg $_{6}-\mathrm{OH} \mathbf{9}$ and clicked product 10 concentration by LC-MS/MS analysis. (c) Blue: plasma concentration of $\mathrm{WN}_{3} \mathbf{2}$. Red: plasma concentration of clicked product 10. (d) Plasma concentration of $\mathrm{BCN}-\mathrm{peg}_{6}-\mathrm{OH}$ 9. These experiments were performed in quadruplicate and error bars indicate s.d.

expected, using the same protocol of injections as for $\mathrm{WN}_{3} 2$ we did not observe any reduction of PT in mice injected with $2 \mathrm{mg} \mathrm{kg}^{-1}$ of Warfarin 1a followed by 20 eq of BCN-peg $6_{6}-\mathrm{OH} 9$, indicating clearly that $\mathrm{BCN}-\mathrm{peg}_{6}-\mathrm{OH} 9$ on its own does not display any pro-coagulant activity (Supplementary Fig. 16). In line with this observation, $\mathrm{BCN}-$ peg $_{6}-\mathrm{OH} 9$ did not display any major effect on Warfarin 1a mediated reduction of clotting factors (Supplementary Fig. 19).

Pharmacokinetics in plasma. We hypothesized that to perform an in vivo reaction the critical point would be the use of a chemical antidote possessing a similar pharmacokinetic profile as the targeted drug. For this reason, pharmacokinetics of $\mathrm{WN}_{3} 2$ and BCN-peg ${ }_{6}-\mathrm{OH} 9$ in living mice were determined using liquid chromatography-mass spectrometry (LC-MS)/MS analysis. $\mathrm{WN}_{3}$ $2\left(2 \mathrm{mg} \mathrm{kg}^{-\mathrm{l}}\right)$ was administered by IP and BCN-peg 6 -OH 9 was delivered by SC injection $\left(52 \mathrm{mg} \mathrm{kg}^{-1}\right.$ corresponding to 20 equimolar quantity with respect to $2 \mathrm{mg} \mathrm{kg}^{-1}$ of $\mathrm{WN}_{3}$ ). Blood samples were collected from the tail vein at selected time intervals $(10,20,30$ and $60 \mathrm{~min})$ and $\mathrm{WN}_{3} 2$ and BCN-peg $-\mathrm{OH} 9$ levels were quantified in plasma preparations using LC-MS/MS analysis. Using this procedure, the maximal plasma concentration of $\mathrm{WN}_{3} 2$ was observed at $10 \mathrm{~min}\left(4.2 \pm 0.4 \mathrm{nmol} \mathrm{ml}^{-1}\right)$ and remained relatively constant for $1 \mathrm{~h}$ (Fig. 5a). This long circulation time may reflect strong binding of $\mathrm{WN}_{3} 2$ to plasma proteins, which was comparable to that observed to non-modified warfarin 1a (97\%, Supplementary Figs 24 and 25). For BCN-peg $6-\mathrm{OH} \mathrm{9,}$ the plasma concentration was the highest between 10 and $30 \mathrm{~min}$ after SC injection, reaching $\sim 200 \mathrm{nmol} \mathrm{ml}^{-1}$ and decreasing more rapidly as compared to $\mathrm{WN}_{3} 2$ (Fig. 5b). Thus, due to $\mathrm{WN}_{3}$ 2 fast uptake into the bloodstream, we decided to inject $\mathrm{BCN}$ peg $_{6}-\mathrm{OH} 95 \mathrm{~min}$ after $\mathrm{WN}_{3} 2$ administration to maximize the time during which the two substrates would be present in the blood at high concentrations.

To address the in vivo formation of the clicked product 10, we first carried out a series of LC-MS/MS analysis of plasma samples from mice following $\mathrm{WN}_{3} 2$ and $\mathrm{BCN}-\mathrm{peg}_{6}-\mathrm{OH} 9$ treatments. To guarantee efficient in vivo reaction, we used 20 equivalents of $\mathrm{BCN}-\mathrm{peg}_{6}-\mathrm{OH} 9$ with respect to $2 \mathrm{mg} \mathrm{kg}^{-1}$ of $\mathrm{WN}_{3} . \mathrm{WN}_{3} 2$ $\left(2.0 \mathrm{mg} \mathrm{kg}^{-1}\right.$ corresponding to $\left.5.7 \mu \mathrm{mol} \mathrm{kg}^{-1}, 1 \mathrm{eq}\right)$ was administered by IP injection whereas $\mathrm{BCN}-$ peg $_{6}-\mathrm{OH} 9$ $\left(52 \mathrm{mg} \mathrm{kg}^{-1}, 114.4 \mu \mathrm{mol} \mathrm{kg}^{-1}, 20 \mathrm{eq}\right)$ was injected SCly $5 \mathrm{~min}$ later, to attain simultaneous maximal $\mathrm{WN}_{3} 2$ and $\mathrm{BCN}-$ peg $_{6}-\mathrm{OH}$ 9 plasma concentration. By choosing a different time point and route of administration for $\mathrm{BCN}-\mathrm{peg}_{6}-\mathrm{OH}$ 9, we aimed to prevent direct contact and reaction between the compounds in the abdomen thereby favoring drug inactivation in the bloodstream. Plasma samples were collected at 10,20,30 and 60 min after the administration of $\mathrm{BCN}-\mathrm{peg}_{6}-\mathrm{OH}$ 9. The clicked product 10 was already detected at a concentration of $3.1 \pm 0.6 \mathrm{nmol} \mathrm{ml}^{-1}$ after $10 \mathrm{~min}$, indicating that the reaction took place in vivo (Fig. $5 \mathrm{c}$ ). Furthermore, inactivated drug $\mathbf{1 0}$ appeared to be the sole product arising from $\mathrm{WN}_{3} 2$ since the sum of the concentration of the remaining $\mathrm{WN}_{3} 2\left(1.0 \pm 0.5 \mathrm{nmol} \mathrm{ml}^{-1}\right.$, Fig. $\left.5 \mathrm{c}\right)$ and the clicked product $10\left(3.1 \pm 0.6 \mathrm{nmol} \mathrm{ml}^{-1}\right)$ corresponds to maximal plasma concentration of $\mathrm{WN}_{3} 2$ expected in absence of $\mathrm{BCN}$ peg $_{6}-\mathrm{OH} 9$ (4.2 $\pm 0.4 \mathrm{nmol} \mathrm{ml}^{-1}$; compare Fig. 5a). Accordingly, the concentration of circulating BCN-peg $6-\mathrm{OH} 9$ was reduced (compare Fig. 5b,d). Most notably, these results also revealed that 


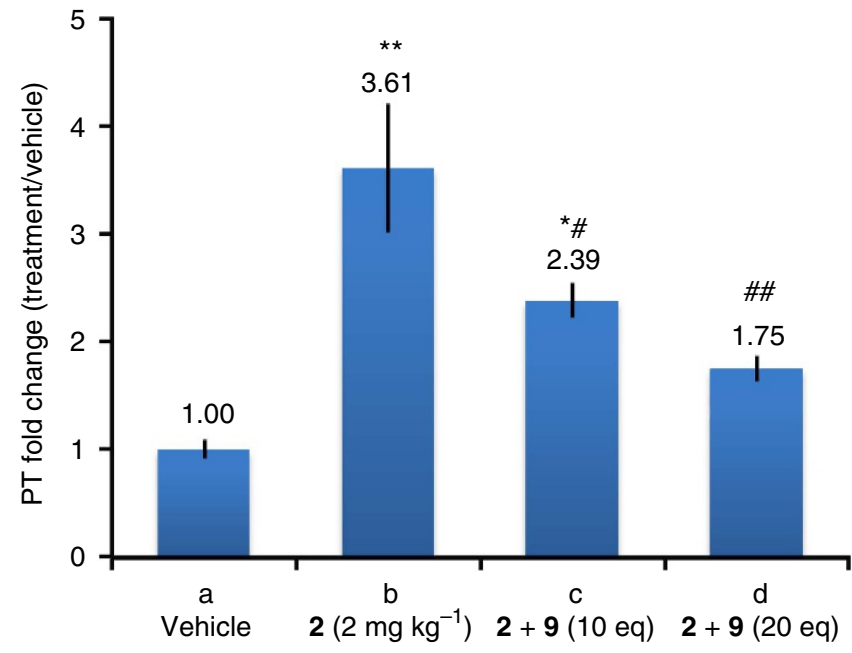

Figure 6 | $\mathbf{W N}_{3}(2)$ biological activity after in vivo click reaction.

Anticoagulant activity represented as a ratio of PT measured for vehicle IP injections. (a) Vehicle IP injections to establish control group of mice $(n=6)$ and used as reference (1.00). (b) Animal treated only with $\mathrm{WN}_{3} 2$ (2.0 $\mathrm{mg} \mathrm{kg}^{-1}, 5.7 \mu \mathrm{mol} \mathrm{kg}^{-1}, 1 \mathrm{eq)}$ twice with a 24 -h gap by IP injection $(n=7)$. (c) $\mathrm{WN}_{3} 2 \mathrm{IP}$ injections ( $2.0 \mathrm{mg} \mathrm{kg}^{-1}, 5.7 \mu \mathrm{mol} \mathrm{kg}^{-1}, 1 \mathrm{eq}$ ) were followed by SC injections of BCN-peg $6-\mathrm{OH} 9\left(26 \mathrm{mg} \mathrm{kg}^{-1}, 57.2 \mu \mathrm{mol} \mathrm{kg}{ }^{-1}\right.$, 10 eq) 5 min post- $W_{3} 2$ delivery $(n=4)$. (d) $W N_{3} 2$ IP injections (2.0 $\mathrm{mg} \mathrm{kg}^{-1}, 5.7 \mu \mathrm{mol} \mathrm{kg}^{-1}, 1 \mathrm{eq}$ ) were followed by SC injections of $\mathrm{BCN}-$ peg $_{6}-\mathrm{OH} 9\left(52 \mathrm{mg} \mathrm{kg}^{-1}, 114.4 \mu \mathrm{mol} \mathrm{kg}{ }^{-1}, 20\right.$ eq) $5 \mathrm{~min}$ post-WN $\mathrm{WN}_{3} 2$ delivery $(n=7)$. Statistical differences identified with PLSD Fischer test were indicated as: ${ }^{\star} P<0.05$ and ${ }^{\star \star} P<0.01$, as compared to vehicle-treated mice and ${ }^{\#} P<0.05$ and ${ }^{\# \#} P<0.01$, with respect to $W N_{3} 2$ treated mice. Error bars indicate s.e.m. values.

the click reaction between $\mathbf{2}$ and $\mathbf{9}$ seemed to trigger quick drug elimination because there was no more free $\mathrm{WN}_{3} 2$ nor click product 10 in plasma 60 min after administration of $\mathrm{BCN}^{- \text {peg }_{6}-}$ $\mathrm{OH} 9$ (Fig. 5c). This is consistent with the pharmacokinetic profile of BCN-peg 6 - $\mathrm{OH} 9$ for which the plasma concentration diminished faster after reaching its peak than does $\mathrm{WN}_{3} 2$ (Fig. 5a,b).

$\mathrm{WN}_{3}$ (2) inactivation by click in vivo. Our data indicate that: (i) clicked product $\mathbf{1 0}$ has no anticoagulant activity and (ii) in vivo click reaction can proceed efficiently and lead to the disappearance of $\mathrm{WN}_{3} 2$ in the plasma. To address the functional relevance of $\mathrm{WN}_{3} 2$ modification by SPAAC in vivo, we studied the anticoagulant activity after two IP injections of $\mathrm{WN}_{3}$ $2\left(2 \mathrm{mg} \mathrm{kg}^{-1}\right)$ with a 24 -h interval and each followed $5 \mathrm{~min}$ later by a SC administration of 10 or 20 equivalents of BCN-peg 6 -OH 9 (26 or $52 \mathrm{mg} \mathrm{kg}^{-1}$ respectively). The analysis of PT performed on plasma samples $6 \mathrm{~h}$ after the last injection revealed that $\mathrm{WN}_{3}$ 2 displayed different anticoagulant activity depending on the presence or not of $\mathrm{BCN}-\mathrm{peg}_{6}-\mathrm{OH} 9$ which was reflected by significant interaction between effects of $\mathrm{WN}_{3} 2$ and $\mathrm{BCN}-\mathrm{peg}_{6}-\mathrm{OH}$ 9 administration $(F[2,29]=3.8, P<0.05$; two-way ANOVA, Fig. 6). The neutralizing effect of $\mathrm{BCN}-\mathrm{peg}_{6}-\mathrm{OH} 9$ was dosedependent $(F[2,15]=5.0, P<0.05$; one-way ANOVA for BCNpeg $_{6}-\mathrm{OH} 9$ activity in $\mathrm{WN}_{3} 2$ treated mice) as 10 equivalent of BCN-peg ${ }_{6}-\mathrm{OH} 9$ reduced $\mathrm{WN}_{3}$ activity (from 3.61 to 2.39 PT fold change), whereas the reduction was even stronger after an injection of 20 equivalent of $\mathrm{BCN}-\mathrm{peg}_{6}-\mathrm{OH} 9$ (from 3.61 to 1.75 PT fold change). Importantly, injection of 20 eq of BCN-peg 6 - OH 9 normalized PT in $\mathrm{WN}_{3} 2$ pre-treated mice $(P=0.1$ not significant as compared to 20 eq of $\mathrm{BCN}-\mathrm{peg}_{6}-\mathrm{OH} 9$ or vehicle injected group), whereas PT observed after similar application of only 10 eq of $\mathrm{BCN}-$ peg $_{6}-\mathrm{OH} 9$ still remained higher than the corresponding control groups $(P<0.05$, as compared to 10 eq of $\mathrm{BCN}-\mathrm{peg}_{6}-\mathrm{OH} 9$ or vehicle group). The significant decrease of the $\mathrm{WN}_{3} 2$ activity following BCN-peg $6-\mathrm{OH} 9$ treatment was the consequence of the in vivo click reaction and did not result from pro-coagulant activity of $\mathrm{BCN}-\mathrm{peg}_{6}-\mathrm{OH}$ 9, as two vehicle or warfarin IP treatments followed by administration of BCN-peg $6^{-}$ $\mathrm{OH} 9$ at 26 or $52 \mathrm{mg} \mathrm{kg}^{-1}$ did not alter PT measures $(P>0.2$ and $P>0.9$ for 10 or 20 eq of $\mathrm{BCN}-$ peg $_{6}-\mathrm{OH} 9$ groups as compared to vehicle treatment group, Fig. 4 and Supplementary Fig. 16). The pro-coagulant effects of $\mathrm{BCN}-$ peg $_{6}-\mathrm{OH} 9$ were comparable to activity of high dose $\left(20 \mathrm{mg} \mathrm{kg}^{-1}\right)$ of vitamin $\mathrm{K} 1$ in $\mathrm{WN}_{3}$ 2 treated animals with respect to both reduction of PT values (Supplementary Fig. 17) and decreased activity of all three clotting factors, II, VII and X (Supplementary Fig. 20).

BCN-peg $\sigma_{6}-\mathrm{OH} 9$ administration delayed by 10 and 30 min can also inactivate $\mathrm{WN}_{3} 2$ with equal efficiency as $\mathrm{BCN}-$ peg $_{6}-\mathrm{OH} 9$ administration at 5 min post- $\mathrm{WN}_{3} 2$ treatment (Supplementary Fig. 17). This observation is important as BCN applied at $30 \mathrm{~min}$ should attain its maximal levels in the organism 10-20 min later, which corresponds to $40-50$ min after $\mathrm{WN}_{3} 2$ administration. We found that at this time point $\mathrm{WN}_{3} 2$ is present not only in plasma but also in target organs such as the liver (Fig. 7), suggesting that BCN-peg 6 -OH 9 may react with $\mathrm{WN}_{3} 2$ and clear it not only from circulation, but also from target organs.

However, the PT measures after neutralization of $\mathrm{WN}_{3} 2$ by BCN-peg ${ }_{6}-\mathrm{OH} 9$ showed a tendency $(\mathrm{P}=0.1)$ to stay above the PT of control mice, which could be due to re-equilibration of $\mathrm{WN}_{3} 2$ into circulation from other tissues such as the liver. To address this important point, we have measured the amount of $\mathrm{WN}_{3} 2$ remaining in the liver after $\mathrm{BCN}-\mathrm{peg}_{6}-\mathrm{OH} 9$ treatment. Accordingly, at $45 \mathrm{~min}$ after $\mathrm{BCN}-$ peg $_{6}-\mathrm{OH} 9$ treatment, there are low but detectable levels of free $\mathrm{WN}_{3} 2$ in the liver; $4.3 \mathrm{nmolg}^{-1}$ as compared to $31.4 \mathrm{nmolg}^{-1}$ observed at the same time point in mice injected with $\mathrm{WN}_{3} 2$ alone (compare 'no click reaction' and 'in vivo click reaction' panels in Fig. 7). These data suggest that the remaining low amount of $\mathrm{WN}_{3} 2$ in the liver after the click reaction account at least in part for the residual anticoagulant activity. However, we cannot exclude re-equilibration of $\mathrm{WN}_{3} 2$ from another compartment. Indeed, the neutralizing strained alkyne was designed to have a fast clearance preventing later reaction with re-equilibrated $\mathrm{WN}_{3} 2$. We thus measured the renal clearance of $\mathrm{WN}_{3} 2, \mathrm{BCN}-$ peg $_{6}-\mathrm{OH} 9$ and clicked product $\mathbf{1 0}$.

Renal clearance of $\mathrm{WN}_{3}$ (2) after in vivo click reaction. To study the fate of $\mathrm{WN}_{3} 2$ following BCN-peg 6 - $\mathrm{OH} 9$ administration, we compared its distribution in three body compartments (plasma, liver and urine) at 50-60 min after single IP administration of $\mathrm{WN}_{3} 2\left(2 \mathrm{mg} \mathrm{kg}^{-1}\right)$ in mice which received $\mathrm{BCN}-$ peg $_{6}-\mathrm{OH} 9$ (single SC injection of $52 \mathrm{mg} \mathrm{kg}^{-1}, 20 \mathrm{eq}$ ) or vehicle at $5 \mathrm{~min}$ after $\mathrm{WN}_{3} 2$ treatment. Two-way ANOVA analyses revealed that $\mathrm{WN}_{3} 2$ distribution in different compartments was significantly different depending on the application of $\mathrm{BCN}-\mathrm{peg}_{6}-\mathrm{OH} 9$ as supported by significant interaction between body compartment and $\mathrm{BCN}-$ peg $_{6}-\mathrm{OH} 9$ application $(F[2,13]=6.27, P<0.05)$. This difference reflects almost complete absence of free $\mathrm{WN}_{3} 2$ in plasma, liver and urine after BCN-peg 6 -OH 9 treatment (Fig. 7). This dramatic reduction of $\mathrm{WN}_{3} 2$ was at the expense of a strong increase of clicked product 10, which was found predominantly in the urine $(95.3 \%$ of total clicked product detected in all three body compartments, respectively). These data indicate rapid renal clearance of clicked $\mathrm{WN}_{3} \mathbf{1 0}$ which might be due to strong endogenous preference for such mode of clearance of unbound BCN-peg 6 -OH 9 as indicated by almost exclusive 


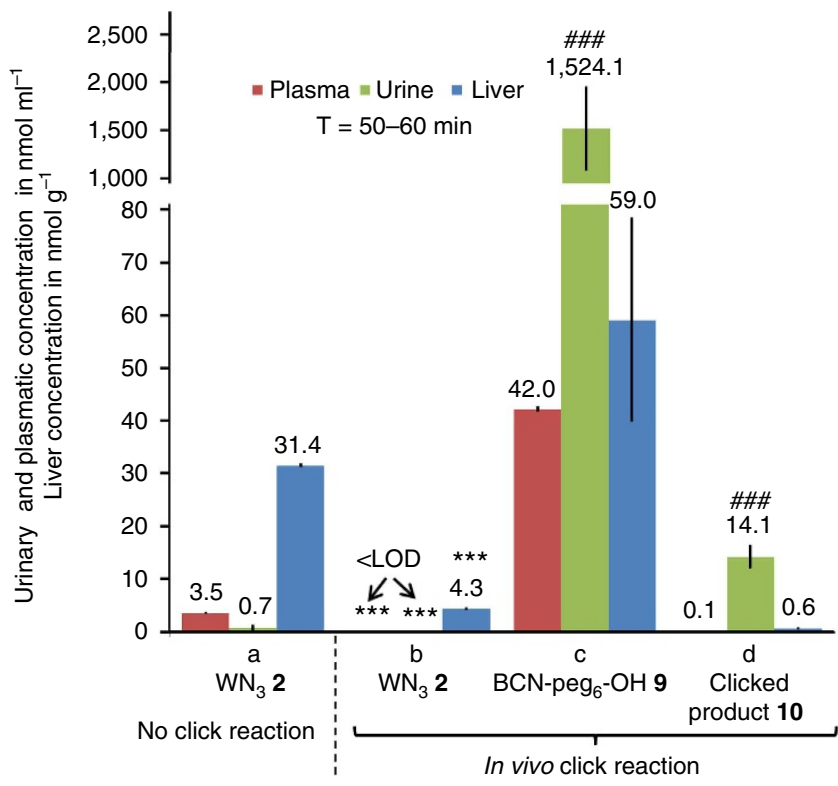

Figure 7 | Effects of in vivo click reaction on $\mathrm{WN}_{3}$ (2) distribution and renal excretion. (a) $\mathrm{WN}_{3} \mathbf{2}$ concentration was measured in the plasma, urine and liver of mice treated with a single dose of $W_{3} \mathbf{2}\left(2.0 \mathrm{mg} \mathrm{kg}^{-1}\right.$, $5.7 \mu \mathrm{mol} \mathrm{kg}^{-1}, 1 \mathrm{eq}$ ) by IP injection $(n=3)$. (b-d) Analyses of free and clicked $\mathrm{WN}_{3} \mathbf{2}$ and $\mathrm{BCN}$-peg ${ }_{6}-\mathrm{OH} \mathbf{9}$ after single IP injection of $\mathrm{WN}_{3} \mathbf{2}$ (2.0 $\mathrm{mg} \mathrm{kg}^{-1}, 5.7 \mu \mathrm{mol} \mathrm{kg}^{-1}, 1 \mathrm{eq}$ ) followed $5 \mathrm{~min}$ later by SC injections of BCN-peg 6 -OH $9\left(52 \mathrm{mg} \mathrm{kg}^{-1}, 114.4 \mu \mathrm{mol} \mathrm{kg}^{-1}, 20\right.$ eq) $(n=3)$.

(a-d) Samples collection have been performed 50-60 min after the last injection. Note that plasma (red) and urine (blue) concentrations of all substrates were reported in $\mathrm{nmol} \mathrm{ml}{ }^{-1}$ whereas liver (green) levels were shown in nmolg ${ }^{-1}$. $<$ LOD (limit of detection), below the detection limit. ${ }^{\star \star \star} P<0.001$ as compared to $W N_{3} \mathbf{2}$ in respective compartments shown in

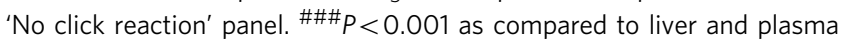
measures of the corresponding compound. Error bars indicate s.e.m.

localization of free $\mathrm{BCN}-\mathrm{peg}_{6}-\mathrm{OH} 9$ in the urine $(93.8 \%$ of all BCN-peg ${ }_{6}-\mathrm{OH} 9$ found in all three body compartments, Fig. 7). The data support the possibility that the efficiency of the click reaction and elimination of clicked product is limited by the time-window of the availability of $\mathrm{BCN}$ due to its fast renal clearance thus preventing neutralization of re-equilibrated $\mathrm{WN}_{3} 2$.

In summary, to investigate the opportunity offered by bioorthogonal chemistry for an in vivo drug neutralization, we designed and synthesized a bioactive analogue of Warfarin 1a bearing an azide $\left(\mathrm{WN}_{3} 2\right)$ and a neutralizing/clearing agent bearing a strained alkyne (BCN-peg $6-\mathrm{OH}$ 9). We confirmed that the product arising from the reaction between $\mathrm{WN}_{3} 2$ and BCNpeg $_{6}-\mathrm{OH} 9$ did not have anticoagulant property. Pharmacokinetics of $\mathrm{WN}_{3} 2$ and $\mathrm{BCN}-\mathrm{peg}_{6}-\mathrm{OH} 9$ were measured to determine the optimum administration conditions for both compounds. Finally, we showed that the treatment of a mouse submitted to anticoagulant therapy $\left(\mathrm{WN}_{3} 2\right)$ with $\mathrm{BCN}-\mathrm{peg}_{6}-\mathrm{OH}$ $\mathbf{9}$ leads to the in vivo formation of the inactivated compound $\mathbf{1 0}$ and total elimination of plasma $\mathrm{WN}_{3} 2$ via renal clearance as demonstrated by LC-MS/MS analysis. Measurement of the PT revealed the concomitant restoration of normal anticoagulant activity. This 'Click \& Clear' strategy opens interesting prospects not only for the development of new drugs with switchable biophysical properties, but also for the design of a universal reversal agent with adequate pharmacokinetic/ biodistribution properties that could neutralize any azide-labelled drug.

\section{Methods}

Animals and in vivo experiments. Young adult C57BL6N male mice (Charles River, France) at 8-12 weeks of age were used throughout this study. All mice were housed in 07:00-19:00 hours light/dark cycle in individually ventilated cages (Tecniplast, Italy). Food and water were freely available. All experiments were approved by local ethics committee and carried out in accordance with the European Community Council Directives of 24 November 1986 (86/609/EEC) and in compliance with the guidelines of CNRS and the French Agricultural and Forestry Ministry (decree 87848).

Formulation of warfarin and $\mathbf{B C N}$ derivatives. Warfarin derivatives (Warfarin $\mathbf{1}$, $\mathrm{WN}_{3} 2$ and clicked product 10) and BCN-peg 6 - OH 9 were solubilized in a vehicle solution of $10 \%(\mathrm{w} / \mathrm{w})$ CDX in PBS and used at $2 \mathrm{ml} \mathrm{kg}^{-1}$ with exception of per os treatments for which Warfarin $1 \mathrm{a}$ or $\mathrm{WN}_{3} 2$ were suspended in $0.5 \%$ methylcellulose solution and used at $10 \mathrm{ml} \mathrm{kg}^{-1}$. In brief, for functional studies, Warfarin 1a or $\mathrm{WN}_{3} 2$ were administrated per os or by IP injection twice with a 24 -h interval and mice were killed for blood collection at different time points after the last injection, as indicated. For $\mathrm{WN}_{3} 2$ inactivation and the corresponding control experiments, 10 or 20 equivalents (with respect to $\mathrm{WN}_{3} 2$ quantity) of BCN-peg ${ }_{6}-\mathrm{OH} 9$ or vehicle were injected subcutaneously 5 min after each of the two $\mathrm{WN}_{3} 2$ or vehicle IP treatments and blood was collected $6 \mathrm{~h}$ after the last injection for prothrombin analysis. All treatments were carried out in the morning between 07:00-09:00 hours. To facilitate comparisons between different experimental conditions, changes of PT measures were reported as fold change with respect to $\mathrm{PT}$ values in control mice, and were calculated as the mean of ratios of each individual PT value from a specific treatment group to the mean PT value of control group.

LC-MS/MS analysis. For pharmacokinetic studies, one treatment was performed followed by repeated blood samples collections from the tail vein at selected time intervals (10, 20, 30 and $60 \mathrm{~min})$ using heparin-coated capillaries. Plasma samples were prepared by centrifugation at room temperature at a minimum of $10 \mathrm{~min}$ after blood collection. This minimal incubation period aimed to reduce an error which could occur at very short periods of incubation and in consequence incomplete plasma preparation. To each $20 \mu \mathrm{l}$ sample of of plasma, $30 \mu \mathrm{l}$ of acetonitrile was added and the samples were centrifuged for $7 \mathrm{~min}$ at 4,000 r.p.m. in order to eliminate proteins. Supernatants were then analysed by LC-MS/MS (Supplementary Fig. 21). For LC-MS/MS analysis of in vivo click reaction, $\mathrm{WN}_{3} 2$ delivery by IP injection was followed $5 \mathrm{~min}$ later by SC injection of BCN-peg 6 - $\mathrm{OH} 9$.

Evaluation of prothrombin time and clotting factors. To evaluate the PT, mice were euthanised by increasing dose of $\mathrm{CO}_{2}$ and blood was collected by intra-cardiac puncture immediately after respiratory arrest. Sodium citrate was added immediately for a final concentration of $1 \%$ and plasma was prepared by centrifugation at room temperature a minimum of $10 \mathrm{~min}$ after blood collection. PT was measured using a Start 4 benchtop hemostasis analyzer and dedicated kit (Neoplastine, Diagnostica Stago) according to manufacture instructions. To evaluate clotting factors we used dedicated kits (Diagnistica Stago). The calibration of the system was performed at the beginning of each analysis using reference plasma samples (Coag Control N + P, Diagnostica Stago) according to manufactures instructions (Supplementary Fig. 18).

Data availability. Data supporting the findings of this study are available within the article and its Supplementary Information files and from the corresponding author upon reasonable request.

\section{References}

1. Bom, A. et al. A novel concept of reversing neuromuscular block: chemical encapsulation of rocuronium bromide by a cyclodextrin-based synthetic host Angew. Chem. Int. Ed. 41, 266-270 (2002).

2. Adam, J. M. et al. Cyclodextrin-derived host molecules as reversal agents for the neuromuscular blocker rocuronium bromide: synthesis and structureactivity relationships. J. Med. Chem. 45, 1806-1816 (2002).

3. Kovac, A. L. Sugammadex: the first selective binding reversal agent for neuromuscular block. J. Clin. Anesth. 21, 444-453 (2009).

4. Yang, L. P. \& Keam, S. J. Sugammadex: a review of its use in anaesthetic practice. Drugs 69, 919-942 (2009).

5. Rusconi, C. et al. RNA aptamers as reversible antagonists of coagulation factor IXa. Nature 419, 90-94 (2002).

6. Heckel, A. et al. An anticoagulant with light triggered antidote activity. Angew. Chem. Int. Ed. 45, 6748-6750 (2006).

7. Rusconi, C. P. et al. Antidote-mediated control of an anticoagulant aptamer in vivo. Nat. Biotechnol. 22, 1423-1428 (2004).

8. Dyke, C. K. et al. First-in-human experience of an antidote-controlled anticoagulant using RNA aptamer technology: a phase 1a pharmacodynamic evaluation of a drug-antidote pair for the controlled regulation of factor IXa activity. Circulation 114, 2490-2497 (2006). 
9. Joachimi, A., Mayer, G. \& Hartig, J. S. A new anticoagulant - antidote pair: control of thrombin activity by aptamers and porphyrins. J. Am. Chem. Soc. 129, 3036-3037 (2007).

10. Wang, J., Cao, Y., Chen, G. \& Li, G. Regulation of thrombin activity with a bifunctional aptamer and hemin: development of a new anticoagulant and antidote pair. Chembiochem. 10, 2171-2176 (2009).

11. Oney, S. et al. Development of universal antidotes to control aptamer activity. Nat. Med. 15, 1224-1228 (2009).

12. Burke, P. A. The ups and downs of clinical translation of new technologies: déjà vu all over again. Mol. Ther. 23, 791-792 (2015).

13. Sletten, E. M. \& Bertozzi, C. R. Bioorthogonal chemistry: fishing for selectivity in a sea of functionality. Angew. Chem. Int. Ed. 48, 6974-6998 (2009).

14. Lim, R. K. V. \& Lin, Q. Bioorthogonal chemistry: recent progress and future directions. Chem. Commun. 46, 1589-1600 (2010).

15. King, M. \& Wagner, A. Developments in the field of bioorthogonal bond forming reactions-Past and present trends. Bioconjug. Chem. 25, 825-839 (2014).

16. Borrmann, A. \& van Hest, J. C. M. Bioorthogonal chemistry in living organisms. Chem. Sci. 5, 2123-2134 (2014).

17. Chang, P. V. et al. Copper-free click chemistry in living animals. Proc. Natl Acad. Sci. USA 107, 1821-1826 (2010).

18. Prescher, J. A., Dube, D. H. \& Bertozzi, C. R. Chemical remodelling of cell surfaces in living animals. Nature 430, 873-877 (2004).

19. Rong, J. et al. Glycan in intact rat hearts and glycoproteomic analysis reveal the upregulation of sialylation during cardiac hypertrophy. J. Am. Chem. Soc. 136, 17468-17476 (2014).

20. Rossin, R. et al. In vivo chemistry for pretargeted tumor imaging in live mice. Angew. Chem. Int. Ed. 49, 3375-3378 (2010).

21. Devaraj, N. K., Thurber, G. M., Keliher, E. J., Marinelli, B. \& Weissleder, R. Reactive polymer enables efficient in vivo bioorthogonal chemistry. Proc. Natl Acad. Sci. USA 109, 4762-4767 (2012).

22. Rossin, R., Läppchen, T., van den Bosch, S. M., Laforest, R. \& Robillard, M. S. Diels-Alder reaction for tumor pretargeting: in vivo chemistry can boost tumor radiation dose compared with directly labeled antibody. J. Nucl. Med. 54, 1989-1995 (2013).

23. Andrew, O. O. \& Herbert, B. C. The anticoagulant activity of some selected warfarin analogues. J. Pharm. Pharmacol. 39, 142-144 (1987).

24. Abildgaard, C. F., Lewis, J. P. \& Harrison, J. Quantitative coagulation studies in mice. Lab. Anim. Sci. 22, 99-101 (1972).

\section{Acknowledgements}

Financial support was provided by the International Center for Frontier Research in Chemistry (icFRC) and the Agence Nationale pour la recherche (ANR). This study was also supported by the grant ANR-10-LABX-0030-INRT, a French State fund managed by the Agence Nationale de la Recherche under the frame program Investissements d'Avenir ANR-10-IDEX-0002-02. We thank Michel Mosser (LFCS-UMR7199) for BCN synthesis, Patrick Gizzi (PCBIS-TechMed) for LC-MS/MS analysis, Nelly Frossard and François Daubeuf (UMR 7200-Laboratoire d'innovation thérapeutique) for helpful discussions of in vivo experiments and formulation for drug treatments. We also gratefully acknowledge Prof. Andrew Griffiths (Laboratory of Biochemistry, ESPCI Paris) for proofreading our manuscript and Dr Jean-Pierre Rameau (Hôpital de Hautepierre) for helpful discussions.

\section{Author contributions}

A.W. and W.K. conceived and designed the project study. M.R. and S.U. synthesized and characterized the molecules studied in this work. W.K., M.R. and S.U. performed the in vivo experiments and/or evaluated anticoagulant activity. A.W., W.K. and S.U. wrote the manuscript.

\section{Additional information}

Supplementary Information accompanies this paper at http://www.nature.com/ naturecommunications

Competing interests: The authors declare no competing financial interests.

Reprints and permission information is available online at http://npg.nature.com/ reprintsandpermissions/

How to cite this article: Ursuegui, S. et al. An in vivo strategy to counteract post-administration anticoagulant activity of azido-Warfarin. Nat. Commun. 8, 15242 doi: 10.1038/ncomms15242 (2017).

Publisher's note: Springer Nature remains neutral with regard to jurisdictional claims in published maps and institutional affiliations.

(c) (i) This work is licensed under a Creative Commons Attribution 4.0 International License. The images or other third party material in this article are included in the article's Creative Commons license, unless indicated otherwise in the credit line; if the material is not included under the Creative Commons license, users will need to obtain permission from the license holder to reproduce the material. To view a copy of this license, visit http://creativecommons.org/licenses/by/4.0/

C) The Author(s) 2017 